\title{
O Lugar do Afeto, o Afeto pelo Lugar: O que Dizem os Idosos? ${ }^{1}$
}

\author{
Danielle Macedo \\ Carolina Vilela Oliveira \\ Isolda de Araújo Günther ${ }^{2}$ \\ Universidade de Brasilia \\ Susana Martins Alves \\ OPENspace Research Centre, \\ Edinburg College of Art, UK \\ Thaís Santos Nóbrega \\ Universidade Federal do Rio Grande do Norte
}

\begin{abstract}
RESUMO - Este estudo investigou os lugares favoritos, os lugares evitados e os afetos a eles relacionados como exemplos de estratégias de regulação das emoções. Um total de 340 participantes (169 homens e 171 mulheres), com idades entre 60 e 90 anos, residentes em Brasília e Natal, responderam individualmente a uma entrevista semi-estruturada, indicando os lugares favoritos quando se sentem alegres e quando não se sentem alegres, os lugares evitados e as razões para tais escolhas. Os resultados apontam preferência por ambientes facilitadores de interação social quando se sentem alegres e, em seguida, a sua própria casa. Essas preferências se invertem, quando não se sentem alegres, sendo indicado também que lugares agitados e cheios de estimulação são evitados. Foram encontradas mais similaridades do que diferenças quanto ao local de residência e poucas diferenças quanto ao gênero. Os resultados são discutidos em termos da perspectiva do curso de vida, bem como da influência recíproca pessoa versus ambiente.
\end{abstract}

Palavras-chave: lugar e auto-regulação emocional; idosos; psicologia do desenvolvimento; psicologia ambiental.

\section{The Place of Affect, the Affect for the Place: What do the Elderly Say?}

\begin{abstract}
This study investigated the favorite and the avoided places, as well as the affects related to them as examples of environmental strategies of emotional regulation. A total of 340 participants (169 male and 171 female), between 60 to 90 years old, living in two Brazilian capitals, Brasília and Natal, responded individually to a semi-structured interview about their favorite places when they are happy and unhappy, the places they avoid and the reasons for these choices. The results point out to preferences, when they are happy, for environments that allow for social interaction and, next, for their own homes. When the respondents are not happy, the order of those preferences is reverse, and they refer also to places that are agitated and full of stimulation as avoided. It was found more similarities than differences with respect to place of residence, and few differences regarding to gender. Results are discussed in terms of the lifespan perspective as well as the reciprocal influence between person versus environment.
\end{abstract}

Keywords: place and emotional self-regulation; elderly; developmental psychology; environmental psychology.

No passado recente, pessoas acima de 60 anos formavam um grupo minoritário da população mundial. A explosão demográfica verificada na segunda metade do Século XX, aliada aos avanços na área da saúde, superou os vaticínios mais otimistas de que os indivíduos podem viver mais e, quem sabe, melhor.

O aumento deste segmento da população tem despertado crescente interesse científico para a compreensão das dimensões do processo de envelhecimento. Apoiando-se em

1 À época da pesquisa, Danielle Macedo e Carolina Vilela Oliveira eram bolsistas do Programa de Iniciação Científica na UnB, Isolda de Araújo Günther era Pesquisadora Associada Sênior no Instituto de Psicologia/UnB, Susana Martins Alves era bolsista recém-doutor do Conselho Nacional de Desenvolvimento Científico e Tecnológico (CNPq) no Departamento de Psicologia/UFRN, e Thaís Santos Nóbrega era bolsista do Programa de Iniciação Científica na UFRN. O trabalho teve apoio do CNPq

2 Endereço: Instituto de Psicologia, Universidade de Brasília. Brasília, DF. CEP 70910-900. E-mail: isolda.gunther@gmail.com. idéias ligadas ao Direito, à Ética, à Psicologia e às noções de bem-estar, conceitos como limites voluntários (Steinberg, 2002), envelhecimento bem-sucedido (Freund \& Baltes, 1998; Khoury, 2005), controle primário e controle secundário (Heckhausen \& Schulz, 1995; Heckhausen, 1997), qualidade de vida (Talbot \& Kaplan, 1991), e resiliência (Couto, Koller \& Novo, 2006; Werner \& Smith, 1992), foram incorporados aos estudos sobre o envelhecimento.

$\mathrm{O}$ que significa neste contexto a palavra lugar? O que buscamos entender ao relacionar lugar, afeto, idoso? As palavras paraíso e inferno, cujos significados estão historicamente ligados à felicidade e ao sofrimento, respectivamente, estão presentes na literatura, na religião e na linguagem do dia-a-dia, expressando os pólos de prazer e desprazer que caracterizam as relações que estabelecemos com o ambiente social e o ambiente físico. O estudo do ambiente social tem sólida tradição na Psicologia (Durkin, 1996; Zastrow \& Kinst-Ashman, 2003). Não se pode afirmar o mesmo sobre o ambiente físico. A Psicologia Ambiental chamou atenção 
para a importância do ambiente físico, incluindo-o em suas teorizações sobre identidade e self. Ao investigar as relações que as pessoas estabelecem com os lugares geográficos, foram associados aos estudos sobre o lugar, conceitos como identidade (Proshansky, Fabian \& Kaminoff, 1983), apego ou vínculo ao lugar (Low \& Altman, 1992) e vinculação aos lugares concatenados (Speller, 2005).

Identidade de lugar é definida por Proshannsky e cols. (1983) como um subsistema da identidade do eu, cuja função consiste em descrever e socializar a pessoa por meio de suas interações com o mundo físico. Os lugares significativos emergem em um contexto social, cultural e econômico, são geograficamente localizados, fornecem aos indivíduos um senso de pertencimento, uma identidade territorial. A identidade de lugar consiste em cognições sobre o mundo físico que podem estar relacionadas à memória, às atitudes, aos valores, às preferências, aos significados e às concepções sobre comportamento e experiência ligados ao cotidiano (Mazumdar \& Mazumdar, 1999; Sommer, 1990).

Bowlby, em sua trilogia Apego e perda (1969), Separação (1973) e Ansiedade e raiva (1980), focalizou suas observações na infância e demonstrou a intensidade da ligação afetiva da criança para com sua mãe; a busca e a manutenção da proximidade física; as reações emocionais de infantes quando separados de suas mães, ou de mães substitutas; a ansiedade de separação quando reunidos, após separação; o desligamento (detachment) afetivo. $\mathrm{O}$ alcance desses estudos foi ampliado por Low e Altman (1992) para além da relação mãe versus criança, sendo denominado apego ou vínculo ao lugar, a relação emocional que as pessoas de qualquer idade estabelecem com os lugares.

O conceito "vinculação aos lugares concatenados" (Speller, 2005, p. 133) diz respeito às fortes ligações que estabelecemos com os espaços dentro e ao redor das nossas casas nas diferentes fases do curso de vida. Esse conceito guarda semelhança com o modelo bioecológico de Bronfenbrenner (Bronfenbrenner \& Morris, 1998), no qual o ambiente é considerado um arranjo de estruturas encaixadas uma dentro da outra, como um conjunto de bonecas russas. Do ponto de vista conceitual, apego ao lugar desponta como um importante componente na constituição do self, contribui para seu desenvolvimento e manutenção, sendo útil na compreensão das relações emocionais que se estabelecem entre a pessoa e o ambiente (Mazumdar \& Mazumdar, 1999).

Os significados dos lugares não são estáticos, constituemse a partir de um processo dinâmico em que são continuamente construídos, desconstruídos e dotados de novos significados. Por fim, os lugares não têm necessariamente o mesmo significado para todas as pessoas (Gustafson, 2001; Purcell, Peron \& Berto, 2001), atuando diferentemente nos pensamentos, nos sentimentos, no estado de humor, nas interações sociais e no bem-estar físico.

\section{Lugares Favoritos e Bem-Estar}

Uma expressão de apego ao lugar é o lugar favorito, aquele no qual a pessoa busca acesso para relaxar, manter-se calma, clarear as idéias, recuperar-se das pressões da vida diária. Especula-se que as razões para buscar o lugar favorito podem estar relacionadas às experiências prazenteiras, mas também ao enfrentamento de situações ameaçadoras ou experiências negativas (Korpela, 2003; Russell \& Snodgrass, 1987). A escolha do lugar favorito é determinada por níveis de bem-estar e pela coerência e compatibilidade com eventos de vida passados ou atuais.

Uma das funções dos lugares preferidos é possibilitar variados tipos de experiências restauradoras, que podem ocorrer tanto em ambientes naturais, quanto em ambientes construídos. $\mathrm{O}$ fato de se estar em um lugar favorito induz mudanças fisiológicas ao proporcionar alterações de humor no sentido positivo, equilibrar a capacidade de atenção e possibilitar maior contemplação dos próprios sentimentos.

Ao tratar das qualidades restauradoras do local em que se mora, assim como da satisfação relacionada a esse local, Kaplan (2001) chama atenção para variáveis que podem ser influenciadas pelo que pode ser visto da janela de casa. As possibilidades de contemplar o céu e o ambiente natural árvores, jardins, praças, parques - pela janela de casa, fazer caminhadas, visitar áreas verdes na vizinhança e trabalhar com jardinagem são importantes preditores de satisfação com o local de residência e das propriedades restauradoras do lar e da vizinhança. Além disso, o fato de os residentes gostarem, ou não, do que podem ver pela janela influencia na sensação de bem-estar.

Em uma revisão da literatura, Newell (1997) constatou que os lugares preferidos geralmente oferecem certo nível de estimulação e que há maior tendência à preferência por ambientes naturais a ambientes urbanos. Ademais, a preferência por ambientes é fortemente influenciada, por exemplo, pela presença, ou não, de vegetação e corpos de água. Os significados dos lugares preferidos, as atividades que neles ocorrem, bem como a percepção de segurança, familiaridade e contato com a natureza foram as justificativas mais citadas para preferência por lugares.

Numa pesquisa sobre lugares favoritos entre adolescentes da Estônia, Sommer (1990) constatou a preferência dos participantes por lugares provedores de oportunidades de contemplação, relaxamento e liberdade de expressão. As características que definiam um lugar como favorito referiamse à capacidade de proporcionar bem-estar, relaxamento, conforto, paz e quietude, sendo que $58 \%$ dos respondentes indicaram preferência por paisagens naturais.

Giuliani (2003) chama atenção para o fato de que sentimentos de afinidade mútua, de comunidade, de fraternidade, bem como de aversão e de hostilidade estão freqüentemente relacionados às questões sobre vínculos a locais ou territórios. Para essa autora, a existência de um vínculo afetivo com os lugares é de extrema importância na qualificação positiva ou negativa de nossa existência. No centro desse processo dinâmico encontra-se o passado ambiental do indivíduo formado pelos lugares, pelos espaços e pelas propriedades que "serviram instrumentalmente para a satisfação das necessidades biológicas, psicológicas, sociais e culturais" (p. 59). Tais reflexões levam Giuliani a salientar a importância da relação entre os conceitos vínculo a lugar e bem-estar.

Estudos sobre bem-estar constituem tema recorrente nas duas últimas décadas (Guedea \& cols., 2006; Xavier \& cols., 2003). A noção de bem-estar é traduzida por meio do conceito de qualidade de vida, por sua vez reconhecidamente 
influenciada por fatores de natureza múltipla e avaliada de forma subjetiva. O componente subjetivo da qualidade de vida é examinado por meio da auto-avaliação. O componente multidimensional requer uma investigação por meio de indicadores objetivos como a habilidade funcional, o bem-estar físico, emocional e social. Quais as contribuições dos estudos empíricos no estabelecimento da relação entre bem-estar, relações sociais e lugar na terceira idade?

O trabalho de Rosel (2003) apresenta um exemplo de pesquisa sobre o tema envelhecer no lugar (aging in place). Essa área de estudos fornece informações sobre as relações com a casa, a vizinhança, a comunidade, enfatiza o valor dos ambientes familiares e investiga o rico conhecimento dos idosos sobre si, sobre as pessoas com as quais convive e compartilha dos mesmos contextos ao longo dos anos. Outro exemplo pode ser visto no trabalho de Lawton (1989), pioneiro no estudo sobre envelhecimento e ambiente, que postulou que o ambiente sócio-físico tem três funções básicas: manutenção, estimulação e apoio. A função de manutenção diz respeito à constância e à previsibilidade do ambiente em termos de satisfação e ao apego aos lugares. A segunda função se refere à existência da estimulação e de seus efeitos nas atividades diárias, nas atividades sociais, no lazer. A função de apoio pode ser vista no potencial do ambiente para compensar competências reduzidas ou perdidas.

Sixsmith e Sixsmith (1991) identificaram o importante papel da casa como localização espacial na velhice. É no espaço da casa que as transições críticas da vida, como a emergência de dificuldades físicas, a viuvez e os problemas com a mobilidade, são re-assimiladas na estrutura da vida das pessoas. Por ser um espaço central, "a casa nossa de cada dia" (Freitag, 2006, p. 25) tem sido referida na literatura como um dos lugares mais apreciados, por propiciar meios para a regulação emocional e para a reflexão sobre a identidade. Segundo Proshansky (1978), as casas são paraísos seguros onde criamos raízes, experimentamos o sentimento de pertença e de identidade com o lugar. Essa, sem dúvida, é uma das razões pela qual a maioria dos idosos refere que gostaria de permanecer em suas casas enquanto for possível (Oswald, Wahl, Mollenkoff \& Schilling, 2000).

Rioux (2005) realizou um estudo com o objetivo de avaliar o bem-estar geral de mulheres idosas que moravam em suas casas, por meio da avaliação do nível de satisfação, do tipo de atividades desempenhadas, dos ambientes e do contexto social. Comprovou o impacto diferencial da idade cronológica, das razões para escolher permanecer em casa, do grau de pressão social percebida incentivando a mudança da residência e do grau de queixas quanto à adequação da casa às contingências da idade. Esse estudo reafirmou ser a casa nosso primeiro universo, por conferirnos privacidade, autonomia, senso de pertencimento, de estabilidade e liberdade de auto-expressão. Por sua vez, Bachelard (2000) afirma que a casa é "nosso canto no mundo" (p. 24), e ainda, "um campo de imagens que dão ao homem razões ou ilusões de estabilidade" (p. 36).

Para Carneiro e Falcone (2004) as possibilidades de uma boa qualidade de vida na terceira idade são maiores quando há o estabelecimento e a manutenção de relações sociais funcionais, importantes para o bem-estar físico e mental e para a maior satisfação pessoal e profissional, caso o idoso ainda desempenhe uma função significativa no trabalho. Assim sendo, uma maior gama de papéis supõe uma maior rede de apoio social e se relaciona positivamente com maiores índices de auto-estima, de habilidades sociais, de assertividade e, negativamente, com neurose, pessimismo e afetos negativos.

Esses dados apontam para algumas questões ligadas ao envelhecimento e para a necessidade de enfrentá-las a fim de possibilitar aos idosos lidar com as próprias transformações físicas, com as mudanças nas redes de apoio social, com as demandas que exigem o ajuste de suas necessidades às condições do ambiente para garantir uma maior satisfação com a vida.

\section{Afeto Negativo e Preferência por Lugar}

Uma dessas questões que tem ligação com os afetos refere-se à tendência a se atribuir aos idosos uma maior propensão para apresentar humor negativo e desenvolver sintomas depressivos. Os achados apresentados na literatura não são consistentes. Por exemplo, Eagles e Whalley (1985) afirmam que os idosos experimentam mais depressão do que as pessoas mais jovens. Já Gatz e Hurwicz (1990) examinaram a presença de sintomas depressivos em 548 pessoas de quatro coortes (20-39, 40-54, 55-69 e 70-98 anos), não encontrando diferença no grau de depressão entre os quatro grupos. Em itens como humor depressivo, dificuldades interpessoais, lentificação locomotora e ausência de bem-estar, o grupo com mais anos de vida obteve escores mais altos no que se refere à ausência de bem-estar. Nos itens relacionados às expectativas quanto ao futuro, os com 70 anos e mais endossaram a ausência de expectativas positivas. Especula-se, no presente artigo, se sentimentos de diminuição de bem-estar motivados pelas perdas sociais ou pelo declínio da saúde podem ser equiparados a sintomas depressivos.

É nesse sentido que Snowdon (2002) mostra que a prevalência de depressão ao redor do mundo, inclusive no Brasil, é de $10 \%$ e argumenta que a saúde física é um fator significativamente associado à depressão e que seus sintomas podem desviar a atenção dos profissionais da saúde, dificultando o reconhecimento e identificação de sintomas depressivos. Snowdon alerta que, além das perdas sociais, é importante reconhecer que o idoso pode apresentar diferentes graus de disfuncionalidade, que por sua vez podem estar ligados às mudanças etárias relacionadas à vascularização do cérebro e podem contribuir para a maior incidência de fatores depressivos. O declínio da saúde pode se estender para a organização cognitiva e diminuir a possibilidade de exploração de novas atividades, inclusive da busca de novos espaços físicos. É possível, portanto, que déficits na memória, na cognição espacial, na diminuição da mobilidade e na capacidade de lidar com novos ambientes interfiram na adaptação e na capacidade de exercer autonomia e, conseqüentemente, na exploração independente de ambientes não-familiares.

A literatura sobre ambientes restauradores não refere o termo depressão, mas humor negativo. Ao estudar a relação entre humor negativo e preferência por lugar em uma amostra de adultos, Korpela (2003) apontou que as pessoas tentam regular ou controlar seu estado emocional escolhendo aonde ir e o que fazer. O estado de humor pode, portanto, 
afetar a escolha de lugares aonde ir e não ir, as atividades a serem desenvolvidas e a decisão de se afastar, ou não, de determinados lugares. É possível que a intenção de planejar uma ida a determinado lugar assuma diferentes significados tais como a melhoria de estados de humor ou de sentimentos negativos, o desejo de alterar o humor negativo para um humor mais positivo, ou a manutenção de estados de humor e sentimentos positivos.

A hipótese de que diferenças de humor influenciam a preferência por lugares em que se pode estar sozinho ou acompanhado é mencionada por Kerr e Tacon (1999). Resultados nessa direção são apresentados por Korpela (2003) que, em um estudo envolvendo participantes entre 20 e 77 anos, indica uma tendência a se escolher como lugar preferido aquele lugar em que se pode estar só, quando se vivencia maiores níveis de humor negativo, porque se espera que ocorra uma melhora do estado de humor depois de visitar o lugar favorito. Adicionalmente, ambientes naturais e a própria residência foram amplamente citados nesses estudos como lugares preferidos, sendo que adultos com altos escores de humor negativo tinham maior tendência a escolher ambientes naturais como lugares preferidos, enquanto que adultos com menores escores de humor negativo tendiam a escolher a própria casa. A pesquisa relata, ainda, que os lugares menos apreciados entre adultos na Finlândia foram, em geral, lugares com tráfego excessivo e com pessoas consideradas desagradáveis, como estações de ônibus e outros meios de transporte, áreas industriais e supermercados.

A teoria e a pesquisa assinalam, portanto, que os lugares significativos emergem em um contexto sociocultural, são geograficamente localizados, fornecem aos indivíduos um senso de pertencimento, uma identidade territorial. Nesse sentido, o conceito de lugar favorito, pelos vínculos e afetos a eles relacionados, por suas propriedades restauradoras (Korpela \& Hartig, 1996), é um conceito importante cuja investigação se torna necessária para o estudo de como as interações entre indivíduos e ambiente se estabelecem (Gifford, 1998). Considerando que o ambiente físico pode ser usado como um mediador para regular as emoções e contribuir para o equilíbrio entre ganhos e perdas e, cientes da necessidade de conhecer dimensões da inter-relação entre o idoso e o ambiente físico, por seu papel regulador nos afetos e sua potencial influência na qualidade de vida dos indivíduos, foi planejado este estudo que tem por objetivo pesquisar as seguintes questões: (a) qual o lugar preferido quando se sente alegre?, (b) por que prefere esse lugar?, (c) qual o lugar preferido quando não se sente alegre?, (d) por que prefere esse lugar?, (e) qual o lugar evitado quando se sente triste?, e (f) por que evita esse lugar? Procurar-se-á identificar possíveis similaridades e diferenças no que se refere ao gênero e à localidade de moradia.

\section{Método}

\section{Participantes}

Participaram deste estudo 340 idosos, 169 (49,7\%) do sexo masculino e $171(50,3 \%)$ do sexo feminino, com idades variando entre 60 e 91 anos. A Tabela 1 apresenta a distribuição da amostra por sexo, idade e localidade.

\section{Instrumento}

Elaborou-se um instrumento na forma de uma entrevista estruturada contendo 10 questões. As questões foram pré-testadas para verificar a adequação da linguagem. $\mathrm{O}$ estudo-piloto indicou que a questão "qual o lugar que você prefere estar quando se sente triste?" deveria ser reformulada para "qual o lugar que você prefere estar quando não se sente alegre?" face ao grande número de participantes que indicava a impossibilidade de responder à questão, uma vez que "nunca se sentia triste" (sic). Além das seis questões do estudo indicadas no final da introdução, foram acrescentados itens relativos às variáveis demográficas.

\section{Procedimento}

Os potenciais participantes foram abordados em pistas de caminhada, feiras, bancos, grupos de convivência de terceira idade e clubes das duas capitais brasileiras. Os pesquisadores os cumprimentavam, explicavam os objetivos da pesquisa, indagavam sobre a disponibilidade e interesse em participar do estudo, indicando que a participação era voluntária e devidamente informada. Após a concordância, foram enunciadas as questões da pesquisa, cujas respostas eram anotadas pelo pesquisador.

Para a análise, procedeu-se à leitura de todas as respostas das entrevistas com a finalidade de realizar uma análise temática. A partir dessa análise, foram derivadas as categorias para codificação das respostas. Para comparar a freqüência das respostas nas diferentes categorias quanto a gênero e localidade utilizou-se a análise de qui-quadrado.

\section{Resultados}

\section{Lugar preferido quando se sente alegre}

Os resultados da primeira pergunta deste estudo: "Qual o lugar que você prefere estar quando se sente alegre?", são apresentados na Tabela 2. As respostas foram agrupadas em quatro categorias: ambiente facilitador de interação social - bares, restaurantes, festas, feiras, shopping $(\mathrm{n}=134)$; a própria casa $(\mathrm{n}=114)$; ambiente natural - chácaras, cachoeiras, fazenda, parques, praia e campo $(\mathrm{n}=46)$ e, finalmente, igreja - templos religiosos de diferentes orientações $(n=46)$. São apresentadas ainda duas comparações: a distribuição por gênero, com as freqüências observadas e as freqüências esperadas considerando a proporção de homens e de mulheres, bem como as freqüências observadas e esperadas no que se refere aos lugares favoritos nas duas localidades. A análise de qui-quadrado revela uma diferença estatisticamente significativa $\left(\chi^{2}=9,93 p=.019\right)$, sendo a fonte desta diferença o maior número de mulheres que mencionou a igreja como o lugar favorito quando se sente alegre, enquanto que maior número de homens indicou a casa. A comparação da distribuição por localidade não apresentou uma diferença estatisticamente significativa $\left(\chi^{2}=1,71, \mathrm{p}=.635\right)$. 
Tabela 1. Distribuição dos participantes por sexo, idade e localidade.

\begin{tabular}{|c|c|c|c|c|c|c|}
\hline & & \multicolumn{4}{|c|}{ Localidade } & \multirow{3}{*}{$\begin{array}{c}\text { Total } \\
\text { N }\end{array}$} \\
\hline & & \multicolumn{2}{|c|}{ Brasília } & \multicolumn{2}{|c|}{ Natal } & \\
\hline & & $\mathbf{N}$ & $\%$ & $\mathbf{N}$ & $\%$ & \\
\hline \multirow{3}{*}{ Sexo } & Feminino & 109 & $55 \%$ & 62 & $43 \%$ & 171 \\
\hline & Masculino & 88 & $45 \%$ & 81 & $57 \%$ & 169 \\
\hline & Total & 197 & $100 \%$ & 143 & $100 \%$ & 340 \\
\hline \multirow{2}{*}{ Idade } & Média & \multicolumn{2}{|c|}{69 anos $91 / 2$ meses } & \multicolumn{2}{|c|}{71 anos 6 meses } & 70 anos 6 meses \\
\hline & Desvio Padrão & \multicolumn{2}{|c|}{6 anos $11 \frac{1}{2}$ meses } & \multicolumn{2}{|c|}{6 anos 2 meses } & 6 anos 8 meses \\
\hline
\end{tabular}

Tabela 2. Lugares favoritos quando o idoso se sente alegre por gênero e localidade.

\begin{tabular}{|c|c|c|c|c|c|c|c|c|c|c|}
\hline \multirow{3}{*}{ Lugares Favoritos } & \multirow{2}{*}{\multicolumn{2}{|c|}{ Total }} & \multicolumn{4}{|c|}{ Gênero } & \multicolumn{4}{|c|}{ Local } \\
\hline & & & \multicolumn{2}{|c|}{ Feminino } & \multicolumn{2}{|c|}{ Masculino } & \multicolumn{2}{|c|}{ Brasília } & \multicolumn{2}{|c|}{ Natal } \\
\hline & $\mathbf{N}$ & $\%$ & $\mathbf{f}_{\text {obs }}$ & $\mathbf{f}_{\text {esp }}$ & $\mathbf{f}_{\text {obs }}$ & $\mathbf{f}_{\text {esp }}$ & $\mathbf{f}_{\text {obs }}$ & $\mathbf{f}_{\text {esp }}$ & $\mathbf{f}_{\text {obs }}$ & $\mathbf{f}_{\text {esp }}$ \\
\hline Ambiente facilitador de interação social & 134 & 39,4 & 72 & 67,4 & 62 & 66,6 & 72 & 77,6 & 62 & 56,4 \\
\hline Casa & 114 & 33,5 & 48 & 57,3 & 66 & 56,7 & 70 & 66,1 & 44 & 47,9 \\
\hline Ambiente natural & 46 & 13,5 & 20 & 23,1 & 26 & 22,9 & 28 & 26,7 & 18 & 19,3 \\
\hline Igreja & 46 & 13,5 & 31 & 23,1 & 15 & 22,9 & 27 & 26,7 & 19 & 19,3 \\
\hline Total & 340 & $99,9 *$ & \multicolumn{2}{|c|}{$171(50,3 \%)$} & \multicolumn{2}{|c|}{$169(49,7 \%)$} & \multicolumn{2}{|c|}{$197(57,9 \%)$} & \multicolumn{2}{|c|}{$143(42,1 \%)$} \\
\hline
\end{tabular}

* soma diferente de $100 \%$ por causa de erro de arredondamento

Tabela 3. Lugares favoritos quando o idoso não se sente alegre por gênero e localidade.

\begin{tabular}{|c|c|c|c|c|c|c|c|c|c|c|}
\hline & \multirow{2}{*}{\multicolumn{2}{|c|}{ Total }} & \multicolumn{4}{|c|}{ Gênero } & \multicolumn{4}{|c|}{ Local } \\
\hline & & & \multicolumn{2}{|c|}{ Feminino } & \multicolumn{2}{|c|}{ Masculino } & \multicolumn{2}{|c|}{ Brasília } & \multicolumn{2}{|c|}{ Natal } \\
\hline & $\mathbf{N}$ & $\%$ & $\mathbf{f}_{\text {obs }}$ & $\mathbf{f}_{\text {esp }}$ & $f_{\text {obs }}$ & $\mathbf{f}_{\text {esp }}$ & $\mathbf{f}_{\text {obs }}$ & $\mathbf{f}_{\text {esp }}$ & $f_{\text {obs }}$ & $\mathbf{f}_{\text {esp }}$ \\
\hline Casa & 211 & 62,2 & 102 & 106,4 & 109 & 104,6 & 120 & 122,6 & 91 & 88,4 \\
\hline $\begin{array}{l}\text { Ambientes facilitadores de } \\
\text { interação social }\end{array}$ & 57 & 16,8 & 30 & 28,8 & 27 & 28,2 & 29 & 33,1 & 28 & 23,9 \\
\hline Igreja & 49 & 14,4 & 33 & 24,7 & 16 & 24,3 & 34 & 28,5 & 15 & 20,5 \\
\hline Ambiente natural & 22 & 6,5 & 6 & 11,1 & 16 & 10,9 & 14 & 12,8 & 8 & 9,2 \\
\hline Total & 339 & $99,9 *$ & \multicolumn{2}{|c|}{$171(50,4 \%)$} & \multicolumn{2}{|c|}{$168(49,6 \%)$} & \multicolumn{2}{|c|}{$197(58,1 \%)$} & \multicolumn{2}{|c|}{$142(41,9 \%)$} \\
\hline
\end{tabular}

* soma diferente de $100 \%$ por causa de erro de arredondamento

\section{Razões para preferência por lugar quando se sente alegre}

"Por que prefere este lugar?" As justificativas para as preferências pelos lugares foram: relacionar-se $(\mathrm{n}=$ $121)$; sentir-se bem $(\mathrm{n}=118)$; lazer $(\mathrm{n}=51)$; religiosidade $(n=22)$; sentir-se autônomo $(n=18)$; conectar-secom a natureza $(\mathrm{n}=10)$. Não foram encontradas diferenças estatisticamente significativas nem quanto a gênero, nem quanto à localidade.

\section{Lugar preferido quando não se sente alegre}

"Qual o lugar que você prefere estar quando não se sente alegre?" Os resultados dessa questão são apresentados na Tabela 3. Permanecer na própria casa foi a resposta mais freqüente $(n=211)$ entre homens e mulheres das duas localidades, sendo quase quatro vezes maior do que a segunda razão mencionada, ambientes facilitadores de interação social $(\mathrm{n}=57)$. Outros lugares mencionados foram igreja $(\mathrm{n}=49)$ e ambiente natural $(\mathrm{n}=22)$. A análise de qui-quadrado mostrou uma diferença estatisticamente significativa $\left(\chi^{2}=10.81, p=0,013\right)$ na comparação por gênero, sendo que a principal contribuição para essa diferença é que "quando não se sente alegre" a igreja foi apontada desproporcionalmente por maior número de mulheres e menor número de homens do que o esperado e ambiente natural foi desproporcionalmente indicado por maior número de homens e menor número de mulheres 
Tabela 4. Lugares evitados quando o idoso se sente triste por gênero e localidade.

\begin{tabular}{|c|c|c|c|c|c|c|c|c|c|c|}
\hline & \multirow{2}{*}{\multicolumn{2}{|c|}{ Total }} & \multicolumn{4}{|c|}{ Gênero } & \multicolumn{4}{|c|}{ Local } \\
\hline & & & \multicolumn{2}{|c|}{ Feminino } & \multicolumn{2}{|c|}{ Masculino } & \multicolumn{2}{|c|}{ Brasília } & \multicolumn{2}{|c|}{ Natal } \\
\hline & $\mathbf{N}$ & $\%$ & $f_{\text {obs }}$ & $f_{\text {esp }}$ & $f_{o b s}$ & $\mathbf{f}_{\text {esp }}$ & $f_{o b s}$ & $\mathbf{f}_{\text {esp }}$ & $f_{\text {obs }}$ & $\mathbf{f}_{\text {esp }}$ \\
\hline Lugar agitado & 116 & 34,5 & 52 & 58,3 & 64 & 57,7 & 53 & 68,0 & 63 & 48,0 \\
\hline Lugar triste & 76 & 22,5 & 34 & 38,2 & 42 & 37,8 & 33 & 44,6 & 43 & 31,4 \\
\hline Não há lugar que evite & 69 & 20,5 & 41 & 34,7 & 28 & 34,3 & 52 & 40,6 & 17 & 28,4 \\
\hline Multidão & 56 & 16,7 & 32 & 28,2 & 24 & 27,8 & 48 & 32,8 & 8 & 23,2 \\
\hline \multirow[t]{2}{*}{ Casa } & 19 & 5,7 & 10 & 9,6 & 9 & 9,4 & 11 & 11,1 & 8 & 7,9 \\
\hline & 336 & $99,9 *$ & \multicolumn{2}{|c|}{$169(50,3 \%)$} & \multicolumn{2}{|c|}{$167(49,7 \%)$} & \multicolumn{2}{|c|}{$197(58,6 \%)$} & \multicolumn{2}{|c|}{$139(41,4 \%)$} \\
\hline
\end{tabular}

* soma diferente de $100 \%$ por causa de erro de arredondamento.

do que o esperado. A comparação da distribuição por localidade não indicou diferença significativa.

\section{Razões para preferência por lugar quando não se sente alegre}

"Por que você prefere este lugar quando não se sente alegre?". As justificativas mais mencionadas foram: voltar a sentir-se bem $(\mathrm{n}=118)$; evitar contato $(\mathrm{n}=70)$; resolver problemas $(\mathrm{n}=52)$; relacionar-se $(\mathrm{n}=33)$; religiosidade $(\mathrm{n}=26)$; lazer $(\mathrm{n}=15)$.

\section{Lugar evitado quando se sentem tristes}

"Qual o lugar que você evita quando está triste?". Os lugares indicados foram: lugar agitado - lugares barulhentos, cheios de estimulação como bares, clubes, festas e reuniões $(\mathrm{n}=116)$; lugar triste - cemitérios, hospitais e lugares com pessoas negativas $(\mathrm{n}=76)$; multidão $(\mathrm{n}=56)$; a própria casa $(\mathrm{n}=19)$. Dentre os respondentes, 69 indicaram que não há lugar que evitem. A comparação da distribuição por localidade mostra uma diferença estatisticamente significativa $\left(\chi^{2}\right.$ $=40,16 ; p=000)$, sendo que a maior contribuição é que, em Brasília, desproporcionalmente maior número de pessoas indicou que evita lugar agitado e lugar triste e, em Natal, desproporcionalmente maior número de pessoas indicou evitar multidão quando se sente triste (vide Tabela 4).

\section{Razões para evitar lugar quando se sente triste}

"Por que você evita este lugar?" As razões indicadas para evitar determinado lugar quando se sente triste foram: sentir-se perturbado $(\mathrm{n}=95)$; não aumentar a tristeza $(\mathrm{n}=$ 93); não afetar o ambiente $(\mathrm{n}=40)$; evitar contato $(\mathrm{n}=34)$. Sessenta e nove respondentes indicaram não evitar nenhum lugar, pois nunca se sentem tristes.

\section{Discussão}

Este estudo tem como fundamento a perspectiva que o desenvolvimento humano é um sistema de interação mútuo, múltiplo e contínuo em todos os seus níveis, do molecular ao cultural, e ilustra uma maneira de estudar a pessoa incluída em seu ambiente. Adotar esse princípio teve como objetivo investigar estratégias ambientais que regulam as emoções por meio de lugares favoritos e evitados. Em outras palavras, buscou-se compreender as manifestações da experiência humana que se expressam na relação afetiva que estabelecemos com os lugares.

Verificou-se que, quando se sente alegre, são preferidos os ambientes facilitadores de interação social. Os relatos mais freqüentes foram: "Conversando com os amigos" (M, 73 anos); "No Shopping, prá ver o pessoal" (F, 75 anos); "Em companhia dos amigos" (F, 80 anos), "Prefiro estar com minha familia, na casa de minha filha" (M, 79 anos). Observa-se nas respostas incluídas nessa categoria um liame entre lugar preferido, atividade desenvolvida - no caso relação social - e afeto, o que está em consonância com os estudos de Korpela (2003), Rioux (2005) e Rosel (2003).

Esse resultado contradiz teorias clássicas sobre o envelhecimento (Cumming \& Henry, 1961; Eisdorfer, 1970) que conferem ao idoso a diminuição da intensidade emocional, o aumento de pensamento auto-centralizado e a tarefa psicológica central de desengajar-se emocionalmente. Entretanto, essa busca pela interação social é consistente com a formulação do $9^{\circ}$ estágio da teoria de Erikson (1998), velhice versus comunidade, especialmente com as observações acrescentadas por Joan Erikson após sua morte. Esta autora destacou que, no diagrama epigenético, em que cada sistema ou órgão tem seu tempo de surgimento, a tarefa desse último estágio consiste no confronto entre retirar-se, isolar-se (velhice) ou envolver-se, interagir (comunidade) e que a força motriz de busca de contato e relacionamento está ligada a conteúdos afetivos (Erikson, 1998).

O segundo lugar indicado como favorito quando se sente alegre foi a própria casa: "Na minha casa" (F, 72 anos); "Na minha casa. É que saio tanto. Passo o dia fora que é bom e feliz chegar bem em casa" (M, 70 anos). Duas outras categorias foram registradas. Uma delas foi a igreja: "Na igreja" (F, 72 anos); "Na igreja. Acho até que quando vou fico mais alegre" (M, 75 anos). A outra foi ambientes naturais " $\mathrm{Na}$ fazenda. É bonito, tem água e animais"(F, 70 anos); "No parque" (M, 66 anos). As razões dadas para esse conjunto de escolhas estão ligadas ao potencial desses ambientes para satisfazer à necessidade de se relacionar, sentir-se bem, 
sentir-se autônomo, vivenciar experiência religiosa, bem como proporcionar tranqüilidade, paz, segurança, diversão e privacidade.

Quando não se sente alegre, contrariamente aos resultados de Korpela (2003), a própria casa foi nomeada por mais da metade dos respondentes. Ambientes facilitadores de interação social foram citados como favoritos na segunda posição. Nesse caso, a interação social se configura como um recurso para enfrentar condição emocional adversa, assim como um estímulo para se distrair da situação problema. São referidos, também, igreja e ambiente natural. As justificativas mais freqüentes para esse conjunto de escolhas foram: voltar a sentir-se bem, evitar contato, resolver problemas e obter conforto por meio da experiência religiosa.

Quando não se sentem alegres evitam lugares agitados, lugares tristes, multidão e a própria casa. Os relatos indicam que esses lugares são evitados porque fazem a tristeza aumentar, provocam perturbação, inquietação, irritação e nervosismo, sugerindo que excedem a capacidade de manejo ambiental, o chamado environmental-fit preconizado por Lawton (1989) e Nahemow (2000). O dado que 6\% dos respondentes evita a própria casa quando não se sentem alegrem, exemplifica a variabilidade intra-individual do desenvolvimento, uma das proposições teóricas da perspectiva do curso de vida (Staudinger, Marsiske \& Baltes, (1995) que parece se acentuar com o passar dos anos. Dentre os respondentes, 69 indicaram não evitar nenhum lugar, pois nunca se sentem tristes.

Dois resultados merecem destaque: (a) o que distingue o papel exercido pela casa, independentemente do estado emocional; e (b) o que diz respeito ao baixo índice de referência à natureza. No primeiro caso, a literatura assinala que adultos com menores escores de humor negativo tendem a escolher a própria casa, por ser um espaço de privacidade, um local onde se pode exercitar a reflexão, manter ou evitar contato, e fortalecer um dos mais fortes vínculos existentes, o vínculo com os familiares. É por meio da família, fonte primária de recursos, que são oferecidos suporte emocional, logística para a vida cotidiana, apoio financeiro e ligação com a comunidade (Korpela, 2003; Proshansky,1978; Rioux; 2005; Sixsmith \& Sixsmith,1991. Marcus (1995) nos lembra que: "a casa preenche muitas necessidades: auto-expressão, um reservatório de memórias, um refúgio do mundo exterior, um casulo onde nos sentimos nutridos e baixamos nossa guarda" (p. 4).

Uma explicação alternativa para os achados referentes à casa diz respeito às perdas físicas e de mobilidade que fazem com que os ambientes fora do lar se tornem cada vez menos acessíveis. Assim, a preferência por estar em casa pode ser resultado da realidade de mudanças, sendo configurada de acordo com a conceituação de Lawton (1989) e Nahemow (2000) como esquiva de ambientes percebidos como excedendo as capacidades dos indivíduos. A familiaridade do lar e a privacidade que ele proporciona podem ensejar maior controle das contingências, possibilitarem consonância entre as necessidades da pessoa e os recursos ambientais para satisfazê-las.

Outro resultado a ser destacado, diz respeito ao baixo índice de referência à natureza encontrado neste estudo. As pesquisas sobre ambientes restauradores as quais tivemos acesso foram desenvolvidas no hemisfério norte.
Seus achados enfatizam as propriedades restauradoras das áreas verdes, dos parques, das paisagens. Esperávamos encontrar, em um país localizado nos trópicos, mais referências à natureza, especialmente em Natal, em face de sua pujança e diversidade ecológica. Será que não vivenciar diferenças sazonais demarcadas, acordar e encontrar o céu azul, e o sol brilhando passam a ser vistos como fenômenos corriqueiros, cenas as quais nos habituamos e deixamos de valorizar?

Articulando os resultados da preferência pela interação social com perspectivas teóricas contemporâneas (Baltes, Lindenberger \& Staudinger, 2006; Staudinger, Marsiske \& Baltes, 1995), salta à vista a propriedade do modelo de seleção, otimização e compensação. A interpretação desses resultados com base nesse modelo sugere que a modalidade interação social é selecionada e otimizada uma vez que essa escolha pode contrabalançar declínios inevitáveis dessa fase do curso de vida e possibilitar recompensas, porque, como afirma Baltes: "contrariamente às concepções tradicionais de desenvolvimento, não há ganho no desenvolvimento sem perda, nem perda sem ganho" (2006, p. 583). Talvez, por meio das interações sociais seja possível continuar a fazer parte da comunidade.

Quanto às diferenças de gênero, as mulheres manifestaram preferência pela igreja e os homens pela casa, quando estão alegres. Ademais, a preferência pela igreja foi reafirmada pelas mulheres quando estão tristes, ao passo que os homens indicaram preferência por ambientes naturais, quando o humor se apresenta triste. Compreende-se que a igreja e os preceitos religiosos a ela relacionados tenham tido uma profunda conexão na vida dessa coorte de mulheres. É curioso verificar, entretanto, que homens, cuja socialização parece se orientar para o mundo exterior, manifestem forte conexão com a casa. Será que ao longo do curso de vida a casa é sentida como Lar, doce lar? Ou será que como resultado da aposentadoria a casa se torna o cerne da identidade e da regulação emocional masculina? Tal resultado se adéqua aos dados da literatura no sentido que adultos com altos escores de humor negativo tendem a preferir ambientes naturais. A preferência por ambientes naturais quando estão tristes, manifestada pelos homens, está em concordância com os resultados dos estudos de Korpela (2003) e Newell (1997), bem como das pesquisas de Kaplan (2001) que atribuem à natureza propriedades restauradoras. No que se refere aos resultados sobre a localidade, não temos dados nem de natureza teórica, nem de natureza empírica para dar significado a esta diferença.

\section{Considerações Finais}

Fazendo uso de uma linguagem concisa podemos afirmar que este trabalho confirmou as funções de manutenção, estimulação e apoio do ambiente sócio-físico postuladas por Lawton (1989), e os seus resultados mostraram uma orientação para o lugar centrado na interação com as pessoas.

As limitações deste estudo se prendem a não inclusão de questões sobre as atividades que as pessoas desenvolvem nos lugares e se ao desenvolver tais atividades preferem estar sós ou na companhia de outras pessoas. Estudos futuros deverão 
incluir e esclarecer essas questões, bem como aprofundar as diferentes dimensões e significados da casa.

Os dados sugerem, em suma, que a história de vida do indivíduo, as suas interações com o ambiente, a qualidade dessas interações e o afeto a elas relacionado influenciam na configuração de um ambiente como preferido ou evitado, que varia de acordo com o estado emocional das pessoas e, tal qual um bumerangue, exerce um impacto emocional sobre as mesmas. A auto-regulação dos afetos é um importante aspecto de nossas vidas pelo papel que exerce na dinâmica intrapsíquica, nas relações sociais, no desempenho cognitivo, na manutenção da saúde física e mental.

\section{Referências}

Bachelard, G. (2000). A poética do espaço. São Paulo: Martins Fontes.

Baltes, P. B., Lindenberger, U. \& Staudinger, U. M. (2006). Life span theory in developmental psychology. Em W. Damon \& R. M. Lerner (Eds.-in-Chief) \& R. M. Lerner (Vol. Ed.). Handbook of child psychology: Vol. I. Theoretical models of human development (6 ${ }^{\mathrm{a}}$ ed., pp. 569-664). New York: Wiley.

Bowlby (1969). Attachment and loss: Vol. 1, Attachment. London: The Hogarth Press and the Institute of Psycho-Analysis.

Bowlby (1973). Attachment and loss: Vol. 2, Separation. London: The Hogarth Press and the Institute of Psycho-Analysis.

Bowlby (1980). Attachment and loss: Vol. 3, Loss. London: The Hogarth Press and the Institute of Psycho-Analysis.

Bronfenbrenner, U. \& Morris, P. A. (1998). The ecology of developmental processes. Em W. Damon (Serie Org.) \& R. M. Lerner (Vol. Org.), Handbook of child psychology: Vol. 1. Theoretical models of human development (pp. 993-1027). New York: Wiley.

Carneiro, R. S. \& Falcone, E. M. O. (2004). Um estudo das capacidades e deficiências em habilidades sociais na terceira idade. Psicologia em Estudo, 9, 119-126.

Couto, M. C. P. de P., Koller, S. H. \& Novo, R. F. (2006). Resiliência no envelhecimento: risco e proteção. In D. V. da S. Falcão \& C. M. de S. B. Dias (Orgs.), Maturidade e velhice: pesquisas e intervenções psicológicas (Vol. 2, pp 315-337). São Paulo: Casa do Psicólogo.

Cumming, E. \& Henry, W. E. (1961). Growing old: The process of disengagement. New York: Basic Books.

Durkin, K. (1996). Developmental Social Psychology. Oxford: Blackwell.

Eagles, J. M. \& Whalley, L. J. (1985). Aging and affective disorders: The age at first onset of affective disorders in Scotland, 19691978. British Journal of Psychiatry, 147,180-187.

Eisdorfer, C. (1970). The implication of research for medical practice. The Gerontologist, 10, 62-67.

Erikson, E. H. (1998). O ciclo completo da vida. Porto Alegre: Artes Médicas.

Freitag, B. (2006, outubro, 8). Casa nossa de cada dia. Correio Braziliense (p. 25).

Freund, A. M. \& Baltes, P. B. (1998). Selection, optimization and compensations strategies of life management: Correlations with subjective indicators of successful aging. Psychology and Aging, 13, 531-543.
Gatz, M. \& Hurwicz, M. L. (1990). Are old people more depressed? Cross-sectional data on Center for Epidemiological Studies depression scale factors. Psychology and Aging, 5, 284-290.

Gifford, R. (1998). Introduction. Journal of Environmental Psychology, 18, 3-4.

Giuliani, M. V. (2003). Theory of attachment and place attachment. In M. Bonnes, T. Lee \& M. Bonaiuto. Psychological theories for environmental issues (pp. 137-170). Great Britain: Ashgate.

Guedea, M. T. D., Albuquerque, F. J. B., Tróccoli, B.T., Noriega, J. A. V., Seabra, M. A. B. \& Guedea, R. L. D. (2006). Relação do bem-estar subjetivo, estratégias de enfrentamento e apoio social em idosos. Psicologia: Reflexão e Crítica, 19, 301-308.

Gustafson, P. (2001). Meanings of place: Everyday experience and theoretical conceptualizations. Journal of Environmental Psychology, 21, 5-16.

Heckhausen, J. (1997). Developmental regulation across adulthood: Primary and secondary control of age-related changes. Developmental Psychology, 33, 178-187.

Heckhausen, J. \& Schulz, R. (1995). A life-span theory of control. Psychological Review, 102, 284-304.

Kaplan, R. (2001). The nature of the view from home. Psychological benefits. Environment \& Behavior, 33, 507-542.

Kerr, J. K. \& Tacon, P. (1999). Psychological responses to different types of location and activities. Journal of Environmental Psychology, 19, 287-294.

Khoury, H. T. T. (2005). Controle primário e controle secundário: relação com indicadores de envelhecimento bem sucedido. Tese de Doutorado, Universidade de Brasília, Brasília.

Korpela, K. (2003). Negative mood and adult place preference. Environment \& Behaviour, 35, 331-346.

Korpela, K. \& Hartig, T. (1996). Restorative qualities of favorite places. Journal of Environmental Psychology, 16, 221-233.

Lawton, M. P. (1986). Environment and aging. Albany, NY: Center for the Study of Aging.

Lawton, M. P. (1989). Three functions of the residential environment. Journal of Housing for the Elderly, 5, 35-50.

Low, S. M. \& Altman, I. (1992). Place attachment: A conceptual inquiry. Em I. Altman \& S. M. Low (Eds.), Place attachment (pp. 1-12), New York: Plenum Press.

Marcus, C. C. (1995). House as a mirror of self. Berkeley, CA: Conari Press.

Mazumdar, S. \& Mazumdar, S. (1999). Women's significant spaces: Religion, space and community. Journal of Environmental Psychology, 19, 159-170.

Nahemow, L. (2000). The ecological theory of aging: Powell Lawton's legacy. Em R. Rubinstein, M. Moss \& M. Kleban (Eds.), The many dimensions of aging (pp. 22-40). New York: Springer.

Newell, P. B. (1997). A cross-cultural examination of favorite places. Environment \& Behaviour, 29, 495-514.

Oswald, F., Wahl, H-W., Mollenkolf, H. \& Schilling, O. (2000). Aging in place in rural areas: Similarities and differences of two settings in East and West Germany. Proceedings of the "Housing in the $21^{\text {st }}$ Century" conference. Sweden: Gävle.

Proshansky, H. (1978). The city and self-identity. Environment and Behavior, 10, 147-183.

Proshansky, H., Fabian, A. K. \& Kaminoff, R. (1983). Placeidentity: Physical world socialization of the self. Journal of Environmental Psychology, 3, 57-83. 
Purcell, T., Peron, E. \& Berto, R. (2001). Why do preferences differ between scene types? Environment and Behavior, 33, 93-106.

Rioux, L. (2005). The well-being of aging people living in their own homes. Journal of Environmental Psychology, 25, 231-243.

Rosel, N. (2003). Aging in place: Knowing where you are. The International Journal of Aging and Human Development, 57, $77-90$

Russell, J. A. \& Snodgrass, J. (1987). Emotion and the environment. Em D. Stokols \& I. Altman (Eds.), Handbook of environmental psychology (Vol. 1, pp. 245-280). New York: Wiley.

Sixsmith, A. \& Sixsmith, J. A. (1991). Transitions in home experience in later life. Journal of Architectural and Planning Research, 8, 181-191.

Snowdon, J. (2002). How high is the prevalence of depression in old age? Revista Brasileira de Psiquiatria, 24 (suppl 1), 42-47.

Sommer, B. (1990). Favorite places of Estonian adolescents. Children's Environments Quaterly, 7, 32-36.

Speller, G. M. (2005). A importância da vinculação aos lugares. Em L. Soczka (Org.), Contextos humanos e Psicologia Ambiental, (pp. 133-167). Lisboa: Fundação Calouste Gulbenkian.

Staudinger, U. M., Marsiske, M. \& Baltes, P. B. (1995). Resilência e níveis de capacidade de reserva na velhice. Em A. L. Neri (Ed.), Psicologia do envelhecimento (pp. 195-228). Campinas: Papirus.
Steinberg, L. (2002). Acerca de los adultos mayores. Actualidad Psicológica, 301, 16-17.

Talbot, J. F., \& Kaplan, R. (1991). The benefits of nearby nature for elderly apartment residents. International Journal of Aging and Human development, 33, 119-130.

Werner. E. E. \& Smith, R. S. (1992). Overcoming the odds: Highrisk children from birth to adulthood. Ithaca, NY: Cornell University Press.

Xavier, F. M. F., Ferraz, M. P. T., Marc, N., Escosteguy, N. U. \& Moriguchi, E. H. (2003). Elderly people's definition of quality of life. Revista Brasileira de Psiquiatria, 25, 31-9.

Zastrow, C. \& Kinst-Ashman (2003). Understanding human behavior and the social environment. Chicago: Wadsworth Publishing.
Recebido em 01.08.2007

Primeira decisão editorial em 01.02.2008

Aceito em 21.05.2008 